\title{
Preventable causative factors leading to hospital admission with decompensated heart failure
}

\author{
A Michalsen, G König, W Thimme
}

\begin{abstract}
Objective-To determine the distribution and importance of various factors, especially the preventable ones, that contribute to cardiac decompensation and subsequent hospital admission for heart failure. Methods-During a one year period patients were prospectively recruited and evaluated during their hospital stay by means of a structured personal interview by trained medical staff and through clinical examination and laboratory investigation.
\end{abstract}

Setting-The cardiological department at a teaching affiliated general community hospital in Berlin, Germany.

Patients-Consecutive sample of 179 patients admitted to hospital with acute decompensation of pre-existing heart failure.

Main outcome measures-Proportional distribution of causative factors leading to hospital admission for heart failure; relative importance of preventable factors; details of patient compliance with diet and medication, and knowledge about medication.

Results-Mean (SD) age was 75.4 (9.9) years. Potential causative factors for decompensated heart failure were identified in $85.5 \%$ of patients. Lack of adherence to the medical regimen was the most commonly identified factor and was regarded as the cause of the cardiac decompensation in $41.9 \%$ of cases. Non-compliance with drugs was found in $23.5 \%$ of patients. Other factors related to hospital admission were coronary ischaemia (13.4\%), cardiac arrhythmias $(6.1 \%)$, uncontrolled hypertension $(5.6 \%)$, and inadequate preadmission treatment $(12.3 \%)$. In all, $54.2 \%$ of admissions could be regarded as preventable.

Conclusions-Many hospital admissions for decompensation of chronic heart failure in patients at a district hospital in Berlin are preventable. Measures are necessary to improve this situation and evaluation of programmes that include patient education, patient follow up, and physician training is needed.

(Heart 1998;80:437-441)

Keywords: chronic heart failure; hospital admission; decompensation

Heart failure is a major and increasing public health problem. At a time when death from coronary artery disease is markedly declining, mortality and morbidity from heart failure is on the increase. ${ }^{12}$ The rate of hospital admission, particularly in industrialised societies with aging populations, has increased progressively over the past years, making heart failure the most common indication for hospital admission in elderly people. ${ }^{3}{ }^{4}$ The costs and politico-sociological implications of this disease are immense and account for a major portion of total inpatient expenditure.

Early readmission of elderly patients with heart failure is common. Rates of readmission range from $27 \%$ to $47 \%$ within three to six months after initial discharge..$^{5-7}$ Investigations carried out in the United States have suggested that most early readmissions are due to behavioural factors that are open to influencemainly inappropriate drug treatment or failure to comply with dietary measures. ${ }^{58} \mathrm{~A}$ further study identified inadequate outpatient medical treatment as a frequent cause of acute decompensation of chronic heart failure. ${ }^{9}$ We were interested to see if these results were applicable in a European country with a different medical system. We prospectively examined the relative importance of various precipitating factors, especially the preventable ones, which led to hospital admission of patients with decompensated chronic heart failure in our cardiology department at Humboldt Hospital, a municipal teaching hospital in Berlin, Germany.

\section{Methods}

Admissions to the department of cardiology, as well as those to the intensive care unit at Humboldt-Krankenhaus, Berlin, were reviewed daily by one of the study investigators. Patients were eligible for the study if they had a previous history and diagnosis of heart failure and if the primary admitting diagnosis was congestive heart failure. All patients were enrolled during a one year period between 1 December 1994 and 30 November 1995. The diagnosis of congestive heart failure was based on finite radiological signs (determined independently by a staff radiologist) in conjunction with typical symptoms (dyspnoea, peripheral oedema). Only patients who had no nurse assistance with their drug regimen were included. We excluded patients with dementia or severe psychiatric illness, as well as primarily intubated patients with anticipated survival of less than a week. Patients who were readmitted to the hospital for congestive heart failure during the study period were included only on the first admission. Overall, 179 patients were included in the study and were asked to their consent to a brief confidential interview during their hospital stay. None of the patients refused. 
Table 1 Characteristics and prescribed drug treatment in 179 patients with decompensated heart failure

\begin{tabular}{|c|c|}
\hline Characteristic & Patients (n) \\
\hline Mean (SD) age (years) & $75.4(9.9)$ \\
\hline Male/female & $93 / 86(52 \% / 48 \%)$ \\
\hline \multicolumn{2}{|l|}{ Aetiology } \\
\hline Coronary artery disease & $120(67.0 \%)$ \\
\hline Valvar disease & $26(14.5 \%)$ \\
\hline Hypertension & $104(58.1 \%)$ \\
\hline Idiopathic cardiomyopathy & $8(3.4 \%)$ \\
\hline Previous admission for heart failure & $130(72.6 \%)$ \\
\hline \multicolumn{2}{|l|}{ Comorbidity } \\
\hline Treated diabetes mellitus & $47(26.3 \%)$ \\
\hline Renal dysfunction ${ }^{\star}$ & $29(16.2 \%)$ \\
\hline \multicolumn{2}{|l|}{ New York Heart Association class } \\
\hline II & $16(8.9 \%)$ \\
\hline III & $59(33.0 \%)$ \\
\hline IV & $104(58.1 \%)$ \\
\hline \multicolumn{2}{|l|}{ Admission chest $x$ ray } \\
\hline Pulmonary oedema & $29(16.2 \%)$ \\
\hline Pulmonary congestion & $150(83.8 \%)$ \\
\hline \multicolumn{2}{|l|}{ Admission physical examination of lungs } \\
\hline No rales or rales $<1 / 2$ lung fields & $28(15.6 \%)$ \\
\hline Rales $>1 / 2$ lung fields & $151(84.4 \%)$ \\
\hline \multicolumn{2}{|l|}{ Drugs prescribed } \\
\hline Digitalis & $117(65.4 \%)$ \\
\hline Loop diuretics & $119(66.4 \%)$ \\
\hline Thiazide diuretics & $38(21.2 \%)$ \\
\hline Total diuretics & $141(78.7 \%)$ \\
\hline Nitrates & $77(43.0 \%)$ \\
\hline ACE inhibitors & $86(48.0 \%)$ \\
\hline$\beta$ Blockers & $12(6.7 \%)$ \\
\hline Calcium antagonists & $60(33.5 \%)$ \\
\hline Antiarrhythmics & $7(3.9 \%)$ \\
\hline Coumarin & $31(17.3 \%)$ \\
\hline \multicolumn{2}{|c|}{ Treatment with digitalis/diuretic/ACE inhibitor } \\
\hline Monotherapy & $43(24.0 \%)$ \\
\hline Double therapy & $71(39.6 \%)$ \\
\hline Triple therapy & $53(29.6 \%)$ \\
\hline None & $12(6.7 \%)$ \\
\hline Mean (SD) sodium (mmol/l) & $139(4)$ \\
\hline \multicolumn{2}{|l|}{ Electrocardiography } \\
\hline Not in sinus rhythm & $59(33.0 \%)$ \\
\hline Current smoker & $19(10.6 \%)$ \\
\hline Heavy alcohol abuse & $2(1.1 \%)$ \\
\hline
\end{tabular}

$\star$ Plasma creatinine $>133 \mu \mathrm{mol} / \mathrm{l}$.

ACE, angiotensin converting enzyme.

All the prestudy standardised interviews were carried out by trained hospital physicians during the hospital stay. Information was obtained on sex, age, smoking habits, number and dates of previous hospital admissions for heart failure, prescribed drugs, and dietary treatment. Specifically questions were asked about the daily intake of fluids, the patient's knowledge of fluid restriction, and whether or not regular weight surveillance was undertaken. Additional questions were asked about salt and alcohol intake, intake of analgesics, and smoking habits. The patients were asked the names and doses of their prescribed drugs and whether they took them regularly. Patients who had basic information about the drug (for example: "Digoxin is for the heart, enalapril for the heart or blood pressure, furosemide is a water pill") were regarded as "informed." Patients who reported taking their drugs only intermittently or not at all were classified as "medication non-compliant." Patients were regarded as "dietary non-compliant" (or untreated) if their daily fluid intake was equal to or more than 2.5 litres per day, or if they regularly salted their food at the table, or both. Heavy alcohol use was defined as five or more drinks a day.

Two of the study investigators reviewed all the medical records to obtain a complete history. Particular attention was paid to the initial physical examination, noting the blood pressure at that time and the laboratory and electrocardiographic findings. Plasma creatine kinase and ECG recordings were obtained at least twice during the first two hospital days.

Patients were then assigned to one of the following subgroups, depending on which factor we saw as the main precipitator of the acute congestion.

(1) Acute coronary ischaemia-We categorised a patient as having acute myocardial infarction when presenting with chest pain, raised serum enzyme levels, and typical ECG changes. Acute coronary ischaemia was also diagnosed if a patient with initial chest pain had progression of ST segment changes suggesting coronary ischaemia, without elevation of creatine kinase.

(2) Arrhythmias - Patients with supraventricular tachycardia and a rapid ventricular rate ( $>150$ beats/min) on admission, or documented recent onset of ventricular or supraventricular tachycardia.

(3) Uncontrolled hypertension-Diastolic blood pressure $\geqslant 105 \mathrm{~mm} \mathrm{Hg}$, despite regular antihypertensive treatment.

(4) Miscellaneous-Patients with heart failure plus other acute systemic illnesses, including pneumonia, renal failure, infective endocarditis, liver disease, or hyperthyroidism.

(5) Non-compliance-All subjects who stopped taking their drugs or took them only intermittently. This subgroup also included patients with excessive fluid intake or excessive sodium intake, or both. Patients were only assigned to this group if they had none of the contributing factors 1 to 4 .

(6) Inadequate treatment before hospital admission-Compliant patients with worsening symptoms of heart failure lasting for more than 24 hours, who, even though they contacted a physician during the acute illness, received no modification of their treatment. Patients were only placed in this group if they had none of the precipitating factors 1 to 5 .

(7) No identified factors - Subjects were placed in this group if they had none of the precipitating factors 1 to 6 .

\section{DATA ANALYSIS}

Data collection and analysis was performed using the Microsoft Excel and SPSS for Windows software. Subgroup analysis of the following variables was carried out: sex, age, comorbidity, and uncontrolled hypertension on admission; we also compared patients who were informed about their prescribed drug treatment with those who were not. Subgroup comparisons were done using contingency tables, $\chi^{2}$ test for dichotomous variables, and two tailed $t$ test for continuous variables.

\section{Results}

The study sample included 86 men and 93 women, mean age 75.4 years, range 49 to 95 . Only 44 patients $(24.6 \%)$ were younger than 70 years. Chest roentgenograms and complete interviews were obtained in all cases. The 
Table 2 Factors related to decompensated heart failure

\begin{tabular}{ll}
\hline Factor & Patients $(n(\%))$ \\
\hline Coronary ischaemia & $24(13.4)$ \\
Cardiac arrhythmia & $12(6.7)$ \\
Uncontrolled hypertension & $21(8.4)$ \\
Miscellaneous causes & $11(6.1)$ \\
& \\
Non-compliance & $77(43.0)$ \\
$\quad$ Dietary sodium excess & $61(34.0)$ \\
$\quad$ Fluid excess $(\geqslant 2.51 /$ day) & $42(23.5)$ \\
$\quad$ Non-compliance with drugs & \\
& \\
Treatment before admission & \\
$\quad$ Symptoms for $>24$ hours and no & \\
modification of treatment at consultation & $52(29.0)$ \\
\hline
\end{tabular}

clinical characteristics of the patients including the prescribed drug treatment are summarised in table 1 . On admission the majority of patients were in New York Heart Association class IV $(55.1 \%)$ or III $(33.0 \%) ; 72.6 \%$ of patients had at least one previous admission for congestive heart failure, mostly within the preceding year.

Twelve patients $(6.7 \%)$ were not being treated with any of the standard heart failure drugs. Only 86 patients $(48.0 \%)$ were treated with angiotensin converting enzyme (ACE) inhibitors, in most cases with enalapril. The prescribed dose per day per patient (mean (SD)) was 8.8 (4.7) $\mathrm{mg}$ for enalapril, 25.6 (14.4) $\mathrm{mg}$ for captopril, 9.5 (1.1) $\mathrm{mg}$ for fosinopril, and 3.5 (1.8) $\mathrm{mg}$ for ramipril. The use and dosage of ACE inhibitors was not correlated with renal dysfunction. Two patients reported termination of ACE inhibitor treatment because of induced cough. Non-steroidal anti-inflammatory drug preparations were used frequently by three patients.

Table 2 shows the precipitating factors related to decompensation of heart failure. Cardiac ischaemia was found in 24 patients $(13.4 \%)$. Cardiac arrhythmias were present in 12 patients $(6.7 \%)$ : sustained ventricular tachycardia in two cases; atrial fibrillation with rapid ventricular rate in nine. One patient had symptomatic bradycardia. Uncontrolled hypertension was identified in 21 patients $(11.7 \%)$. In this subgroup, 11 patients were noncompliant with respect to their drug treatment. Miscellaneous causes were detected in 11 patients $(6.1 \%)$. Pneumonia was present in six patients, among whom was one patient with pneumonia secondary to pulmonary artery embolism. Acute renal failure was present in two patients. Hyperthyroidism was detected in two patients, although systematic screening for

Table 3 Patient information about prescribed drugs

\begin{tabular}{lll}
\hline Drug & Patients $(n)$ & $\begin{array}{l}\text { Patients informed } \\
(n(\%))\end{array}$ \\
\hline Frusemide & 119 & $96(80.6)$ \\
Digitalis & 117 & $85(72.6)$ \\
Thiazide & 38 & $27(71.0)$ \\
ACE inhibitor & 8 & $48(55.8)$ \\
Calcium antagonists & 60 & $42(70.0)$ \\
Nitrates & 77 & $52(67.5)$ \\
$\beta$ Blockers & 12 & $8(66.6)$ \\
Aspirin & 70 & $49(70)$ \\
Coumarin & 31 & $28(90.3)$ \\
Glibenclamide & 28 & $25(89.2)$ \\
Theophylline & 14 & $12(85.7)$ \\
\hline
\end{tabular}

ACE, angiotensin converting enzyme.
Table 4 Causes of acute decompensation

\begin{tabular}{ll}
\hline Factor & $\begin{array}{l}\text { Patients } \\
(n(\%))\end{array}$ \\
\hline Non-compliance with drugs or diet & $75(41.9)$ \\
Cardiac ischaemia & $24(13.4)$ \\
Inadequate preadmission treatment & $22(12.3)$ \\
Cardiac arrhythmia & $11(6.1)$ \\
Miscellaneous factors & $11(6.1)$ \\
Uncontrolled hypertension & $10(5.6)$ \\
No definite factor & $26(14.5)$ \\
\hline
\end{tabular}

this comorbid factor was not performed. Two patients showed severe deterioration of aortic valve stenosis, in one case with concomitant hyperthyroidism.

Forty two patients $(23.5 \%)$ reported taking their drugs only intermittently or having stopped taking them altogether. There were no significant sex or subgroup differences relating to adherence to medical and dietary treatment. In two patients heavy alcohol intake was noted; these were both non-compliant with respect to drug treatment. The non-compliant patient group tended to be younger than the compliant group (72.2 (10.5) v 77.4 (9.7) years; NS) and to have a greater number of prescribed drugs (4.0 (1.5) v 3.7 (1.4); NS). From the interviews we were able to estimate the daily fluid intake quantitatively in 165 patients $(92.2 \%)$. Fluid excess, with a daily intake of 2.5 litres or more, was present in 61 patients $(34.0 \%)$. Only 47 patients $(26.3 \%)$ were aware of the need for fluid restriction or paid attention to their daily fluid intake. Although 155 patients (86.6\%) had scales at home, only 67 patients $(38.2 \%)$ weighed themselves regularly.

One hundred and twenty nine patients $(72.1 \%)$ reported visiting their physician at least once a month, and $141(78.8 \%)$ recognised symptoms of worsening heart failure (dyspnoea in most cases) over a period of more than 24 hours before admission. Although 102 of these patients with worsening symptoms sought out their physician during the phase of deterioration, medical treatment was modified in only 50 of them at the time of consultation.

Table 3 shows the numbers and percentages of patients who were informed/not informed about their prescribed drug treatment. Ninety nine patients $(55.3 \%)$ were correctly informed about their prescribed cardiac drugs. Patients showed the highest level of understanding for coumarin and frusemide. However, their knowledge was not significantly correlated with their compliance with drug treatment. Of the 42 non-compliant subjects, $20(47.6 \%)$ were uninformed about at least one drug; among the 137 medically compliant subjects, $60(43.8 \%)$ were uninformed about at least one prescribed drug (NS).

Overall, potential precipitating factors for congestive heart failure could be identified in 153 patients $(85.5 \%)$. The final categorisation of patients with respect to the most important factor for the acute decompensation of chronic heart failure is shown in table 4. Noncompliance with diet or drug treatment was the most commonly identified factor. Among the 21 patients with uncontrolled hypertension, 11 were non-compliant with their drug treatment 
and were classified in the non-compliance group. Two of the 12 patients who were not being treated with digitalis, diuretics, or ACE inhibitors had no other potential causative factor for their acute decompensation and were classified as inadequately treated.

\section{Discussion}

The assessment and prevention of factors that precipitate acute decompensation in patients with chronic heart failure is an important objective in the care and management of such patients. Until now little research has been published about such precipitators. Those investigations that have been carried out are often dated. ${ }^{10} 11$ Our study showed that noncompliance with drug treatment and diet was the leading precipitating factor for hospital admission with decompensation of chronic heart failure, followed by cardiac ischaemia and inadequate medical treatment. Therefore $54.2 \%$ of admissions should be regarded as probably preventable.

Few studies that deal with precipitating factors for cardiac decompensation specifically address non-compliance with prescribed treatment regimens. In Ghali's investigation, poor compliance was identified as the most common cause of decompensation even more often than in our study $(64.4 \%$ of patients overall; $42.5 \%$ medically non-compliant), followed by uncontrolled hypertension (43.6\%) and cardiac arrhythmias $(28.7 \%)$ in a study of 101 patients. ${ }^{8}$ The patients evaluated in that study came mainly from a poor, working class population in Chicago, Illinois, and had a mean age of 59 years. Cardiac arrhythmia was not so strictly defined as in our study. In a smaller study with a different patient population (mean age 76 years) by Wagdil and coworkers in a district hospital in Zürich, Switzerland, noncompliance was found to be as prevalent as in our study ( $47 \%$ of patients overall; $25 \%$ medically non-compliant). ${ }^{9}$ Neither study gave a quantitative estimate of daily fluid intake, a critical factor in severe heart failure. In our study one third of the patients had a fluid intake of 2.5 litres or more a day, and most patients were not informed about the need to restrict fluid intake.

Our data are limited in part by the subjective method of determining whether readmissions were preventable. Criteria for identifying preventable readmissions have not been defined. Nonetheless in our study the causative factors were classified so as not to overestimate the preventable ones.

As in Ghali's and Wagdil's studies, we assessed non-compliance by means of a standardised interview, which was validated in a pilot phase. Although this method is relatively subjective, it has already been shown that the patient's self report, obtained in a structured interview, correlates well with a pill count assessment. ${ }^{12}$ Since objective methods yield a higher incidence of non-compliance, it is unlikely that non-compliance has been overestimated in our study. Using the complete prescription claims file of a Medicaid programme, Monane et al were able to show a high degree of non-compliance in an elderly study cohort of 7247 subjects with newly initiated digoxin treatment. During the 365 days of follow up these patients were without digoxin for an average of 111 days. Only $10 \%$ of the population filled enough prescriptions to have received adequate daily heart failure treatment. ${ }^{13}$

In a recent prospective study on highly selected patients (mean age 59 years) who were referred for assessment of indications for heart transplantation, poor compliance with the drug treatment regimen was noted in only $15 \%$ of patients with acute decompensation. Here cardiac arrhythmia was the leading precipitating factor. ${ }^{14}$ In addition to the differences in the groups that were studied, these results may reflect the specialised care that was given to these patients, who were part of a special heart failure programme. All patients received comprehensive information and case management by nurses and psychologists. Physicians referring patients were kept constantly updated.

In our study, patients who were compliant tended to be older than those who were not compliant. In the study by Monane et al, age was also significantly associated with better compliance. Surprisingly, in our study the patients' knowledge about their drug treatment was not associated with better compliance. Earlier investigations in patients with chronic illnesses emphasised that increased knowledge alone was not associated with concomitant changes in health behaviour. ${ }^{15}$ Other studiesfor example, of self medication programmes, have shown that intensifying patient education and increasing patient responsibility result in better compliance. ${ }^{16}$

We noted that prescribed treatment on admission was often suboptimal. ACE inhibitors were underused. They were mostly not used in doses sufficient to match those used in the large clinical trials and currently recommended for heart failure treatment. ${ }^{17}{ }^{18}$ In a large cross sectional study of more than 4600 patients admitted to hospital for heart failure in Canada during 1992 and 1993, only slightly more than half of the patients $(53 \%)$ received ACE inhibitors during their admission; such drugs were also used less often in patients aged 70 years and older. ${ }^{19}$ Calcium antagonists were used in about one third of our patients. It is possible that these drugs may in some cases have contributed to the clinical deterioration. Surprisingly more than $20 \%$ of the patients did not receive a diuretic. These data suggest room for improvement in the drug treatment of chronic heart failure.

As in the data of Wagdil et $a l,{ }^{9}$ ischaemia was noted in about $13 \%$ of our patients. According to the defined criteria, cardiac arrhythmias were considered to be the causative factor in cardiac decompensation in only $6 \%$ of our patients. In the studies by Ghali and Wagdil, cardiac arrhythmias were recognised more often, but the criteria for their classification as precipitating factors were not strictly defined. For example, long standing atrial fibrillation with a normal ventricular rate could have been counted as a precipitant in those investigations. In our study, 
chronic supraventricular arrhythmias were only regarded as causative when there was a documented rapid ventricular rate $(>150 / \mathrm{min})$. Observation of a close concordance between arrhythmias and heart failure does not prove a causal link. Arrhythmias may also be an effect of decompensation from another cause.

A limitation and possible bias of our study is the lack of data on ventricular function and the relative importance of diastolic and systolic function. Echocardiography was done in about half of our patients before or during their hospital admission. Because the assessment was not standardised, these data were excluded from further analysis. In the study by Vinson $e t$ al there was a trend towards more frequent readmission in patients with a lower ejection fraction. ${ }^{6}$

We found that nearly $80 \%$ of patients had experienced dyspnoea and oedema for more than 24 hours before admission. Most patients had bathroom scales but did not weigh themselves regularly. In a recent study on 181 older adults admitted for heart failure, the average duration of acute dyspnoea before admission was approximately 24 hours and the duration of oedema and cough was 12 to 14 days. ${ }^{20}$ Regular weight control, improved recognition of the symptoms of heart failure, and earlier medical help could therefore result in fewer hospital admissions.

Our findings suggest that despite progressive symptoms patients often do not obtain prompt and adequate treatment. We did not contact the referring physicians to assess the patients' preadmission treatment. The importance of inadequate outpatient treatment in various populations needs to be evaluated further.

Overall, it seems that in many cases the recurrence of chronic heart failure and the need for readmission to hospital are attributable to preventable factors and not to the underlying disease. Few studies have examined the impact of multidisciplinary programmes involving home care, patient education, and physician training. There is preliminary evidence that such programmes can improve both the quality of treatment and the quality of life and thereby reduce the overall cost of health care in the growing number of elderly patients with congestive heart failure. Recently, Rich et al showed that a nurse directed multidisciplinary intervention during a follow up period of 90 days can significantly reduce the number of hospital admissions and improve the quality of life in elderly patients with heart failure. ${ }^{21}$ However, the effect of such programmes may not be directly translatable from one country to another. Further trials and evaluation of such programmes in different populations with different health care systems are therefore needed. In the light of the magnitude of non-compliance in patients with heart failure, it is difficult to understand why so little attention is paid to this problem in clinical practice and in current therapeutic trials.

We are indebted to $M$ Cronin for her editorial assistance.

1 Kannel WB, Ho K, Thom T. Changing epidemiological features of cardiac failure. Br Heart f 1994;72(suppl):S3-9.

2 Schocken DD, Arrieta MI, Leaverton PE, et al. Prevalence and mortality rate of congestive heart failure in the United States. $\mathcal{F}$ Am Coll Cardiol 1992;20:301-6.

3 McMurray J, McDonagh T, Morrison CE, et al. Trends in hospitalization for heart failure in Scotland 1980-1990. Eur Heart $\mathcal{F} 1993 ; 14: 1158-63$.

4 Ghali JK, Cooper R, Ford E. Trends in hospitalization rates for heart failure in the United States 1973-1986. Arch Intern Med 1990;150:769-73.

5 Gooding J, Jette AM. Hospital readmissions among the elderly. F Am Geriatr Soc 1985;33:595-601.

6 Vinson JM, Rich MW, Sperry JC, et al. Early readmission of elderly patients with congestive heart failure. $7 \mathrm{Am}$ Geriatr Soc 1990;38:1290-5.

7 Krumholz HM, Parent EM, Tu N, et al. Readmission after hospitalization for congestive heart failure among Medicare beneficiaries. Arch Intern Med 1997;157:99-104.

8 Ghali JK, Kadakia S, Cooper R, et al. Precipitating factors leading to decompensation of heart failure: traits among urban blacks. Arch Intern Med 1988;148:2013-16.

9 Wagdil P, Vuilliomenet A, Kaufmann U, et al. Ungenügende Behandlungsdisziplin, Patienteninformation und Medikamentenverschreibung als Ursachen für die Notfallhospitalimentenverschreibung als Ursachizienten Patienten. Schweiz Med Wochenschr 1993;123:108-12.

10 Flint FJ. The factor of infection in heart failure. BMF 1954; 2:1018-22.

11 Carder SL. Precipitating factors in congestive heart failure. f Kansas Med Soc 1967;68:372-6.

12 Haynes RB, Taylor DW, Sackett DL, et al. Can simple clinical measurements detect patient noncompliance? Hypertension 1980;2:757-64.

13 Monane M, Bohn RL, Gurwitz JH, et al. Noncompliance with congestive heart failure therapy in the elderly. Arch with congestive heart failure
Intern Med 1994;154:433-7.

14 Opasich C, Febo O, Ricardi PG, et al. Concomitant factors of decompensation in chronic heart failure. Am $\mathcal{f}$ Cardiol 1996;78:354-7.

15 Padgett D, Mumford E, Haynes M, et al. Meta analysis of the effects of educational and psychosocial intervention on management of diabetes mellitus. F Clin Epidemiol 1988;41: 1007-30

16 Lowe CJ, Raynor DK, Courtney EA, et al. Effects of self medication programme on knowledge of drugs and compliance with treatment in elderly patients. $B M \mathcal{F}$ 1995;310:1229-31.

17 Clark AL, Coats AJS. Severity of heart failure and dosage of angiotensin converting enzyme inhibitors. BMF 1995;310: 973-4.

18 Baker DW, Konstam MA, Bottorff M, et al. Management of heart failure. I. Pharmacologic treatment. $\mathscr{f} A M A$ 1994; 272:1361-6.

19 Clinical Quality Improvement Investigators. Mortality risk and patterns of practice in 4606 acute care patients with congestive heart failure. The relative importance of age, sex, and medical therapy. Arch Intern Med 1996;156:1669-73.

20 Friedman MM. Preadmission symptoms in older adults admitted for heart failure [abstract]. Circulation 1995; 92(suppl I):248

21 Rich MW, Beckham V, Wittenberg C, et al. A multidisciplinary intervention to prevent the readmission of elderly patients with congestive heart failure. N Engl f Med 1995; 333:1190-5 